\title{
Chief urology resident management of the urinary tract in stable patients with high spinal cord injuries - survey results and applications in the era of Competence by Design
}

James Wilson, Avril Lusty

Department of Urology, Queen’s University, Kingston, ON, Canada

Cite as: Can Urol Assoc J 2018 December 3; Epub ahead of print. http://dx.doi.org/10.5489/cuaj.5570

Published online December 3, 2018

***

\begin{abstract}
Introduction: The urologist's role in the management of patients with spinal cord injury (SCI) is to prevent upper tract damage and renal failure while facilitating acceptable means for urine elimination. Residency provides the framework to manage SCI patients. The purpose of this study was to determine the surveillance practices of chief urology residents in high SCI patients (T4/5 and above) and their confidence in managing this patient population.
\end{abstract}

Methods: A 14-question survey was administered at the Canadian chief resident preparation examination in 2017. Questionnaire domains included: visit frequency, imaging modality, laboratory testing, and procedures related to upper and lower tract surveillance.

Results: All 33 candidates completed the questionnaire. Chief residents encountered high SCI patients in either diverse clinical settings (48\%) or solely as hospital inpatients (33\%). Candidates had similar surveillance algorithms for stable high SCI patients. Responses for surveillance cystoscopy in stable high SCI patients varied. When asked how comfortable residents were managing high SCI patients, $42 \%$ responded they were comfortable, while the rest responded neutral, uncomfortable, or very uncomfortable.

Conclusion: Most chief residents made similar surveillance decisions for high SCI patients. Residents did differ on the frequency of cystoscopy and how comfortable they were managing this patient population. In the era of competence by design, this information can be used to highlight training opportunities. 


\section{Introduction}

Urologists have long been a part of the healthcare team for patients with high spinal cord injuries (SCI). In Canada, there are approximately 1400 new traumatic SCIs per year and approximately 2900 new non-traumatic SCIs per year. ${ }^{1}$ This patient populations' interaction with the healthcare system is frequent and their needs are multi-disciplinary. The common indications for encounters with urologists include voiding dysfunction management, urinary tract infection (UTI), stones, fertility and more. ${ }^{2}$ Urologists are skilled at managing these genitourinary concerns. Previous studies have been completed to assess the urologist's practice patterns for the surveillance and management of patients with spinal cord injury; however this older data did not examine the resident perspective. ${ }^{3}$ Currently there are few international guidelines focused on the management of the SCI population and, at this time, the Canadian Urological Association is in the process of developing guidelines from the Canadian perspective. ${ }^{4,5}$

Traditionally the Royal College of Physicians and Surgeons required urology residents to be clinical experts in the category of voiding dysfunction due to neurourologic disease. ${ }^{6}$ Academic urology programs provide the accredited framework to achieve these competencies in a variety of clinical settings. There is significant variability amongst programs as to how these competencies are acquired. The landscape of urology education and resident assessment however is changing. Competency based residency education (Competence by Design, CBD) was implemented for Urology in July 2018. CBD is an evolution of the current Canadian resident training model that ensures competence while addressing gaps in knowledge and promoting accountability and transparency. ${ }^{7}$ Canadian academic urology programs have adopted this CBD model and will ensure the urology resident establishes management practices for the high SCI patient with this new framework in mind. In the era of modern urology training programs and new CBD training initiatives, the resident's knowledge and familiarity with high SCI patients, a well-known vulnerable patient population, should be addressed.

In this study, we aimed to determine the landscape of the urology residents' surveillance and management for the stable high SCI patient and how this can be used in the age of CBD.

\section{Methods}

A 14-question survey was administered to all 2017 Canadian chief urology residents at a national preparation examination. The QUEST examination (Queen’s Urology Examination Skills Training), is a mock examination following the Royal College format and is held annually in Kingston, Ontario. Completion of the survey was voluntary and did not affect their academic standing or QUEST results. The study was approved by Queen's University Research Ethics Board.

The high SCI patient, above T4/5, was used as a focus for chief urology residents to draw their attention to a specific patient population with, not only neurogenic voiding dysfunction, but the further compromise of the upper extremities and all the challenges and potential complications that are associated with this presentation. Questions included where residents 
encountered high SCI patients, and what their routine practices were for: visit frequency, laboratory investigations and their frequency, and preferred imaging modalities and their frequency, all in the setting of stable high SCI patients. They were also asked whether they would treat asymptomatic UTIs in men and women, their antibiotic of choice for symptomatic UTIs and duration of treatment. Chief urology residents were also asked how frequently they would perform urodynamics and routine cystoscopy. Their final question asked how comfortable they were managing this patient population.

The data collected from this survey was intended for descriptive statistics as there was no control group or follow-up surveys.

\section{Results}

All 33 chief urology residents completed the survey. Residents encountered high SCI patients in either diverse clinical settings (48\%) or only as hospital inpatients (33\%) (Table 1). All residents replied they would not treat asymptomatic UTIs in high SCI patients with either indwelling Foley catheters or in those who performed clean intermittent catheterizations. When asked the duration of antibiotics for a symptomatic UTI in women, $64 \%$ responded they would prescribe a 7-day course and $24 \%$ stated they would prescribe a 3-day course of antibiotics. When asked the duration of antibiotics for a symptomatic urinary tract infection in men, $67 \%$ would prescribe a 7 -day course and $15 \%$ would prescribe a 10-day course of antibiotics. When asked which antibiotics they would prescribe for a symptomatic UTI, 55\% chose a fluoroquinolone and $45 \%$ chose sulfa/sulfa-trimethoprim. 73\% of residents would perform routine clinic follow-up annually and $21 \%$ would perform routine clinic follow-up every six months. When asked which test they would use to monitor kidney function, $73 \%$ would use serum creatinine and $21 \%$ would use creatinine clearance. $85 \%$ of residents stated they would monitor kidney function annually, while $9 \%$ and $6 \%$ would monitor kidney function every six months or every two years, respectively. When asked which imaging modality they would use to monitor the upper urinary tracts, $100 \%$ of residents would use ultrasound. $70 \%$ would monitor the upper urinary tracts annually and $24 \%$ would monitor every two years. When asked how frequently they would perform surveillance urodynamics, $55 \%$ responded as needed, and $27 \%$ stated on a regular basis but less frequently than every two years. When asked how frequently they would perform surveillance cystoscopy, 30\% responded annually, $21 \%$ responded every two years, $30 \%$ stated less frequently and $18 \%$ responded no routine schedule but as needed. The final question asked how comfortable residents were managing high SCI patients and $42 \%$ responded they were comfortable, $36 \%$ responded neutral, $18 \%$ responded uncomfortable and $3 \%$ responded very uncomfortable.

\section{Discussion}

Urologists are one of the many healthcare professionals involved in caring for patients with high SCI. High SCI patients can develop significant urologic complications and understanding the 
need for routine follow-up for this patient population, in addition to being competent treating urologic complications, is essential during urology resident training. ${ }^{2}$ With varied exposure amongst urology residents to the high SCI patient many questions yielded very similar responses, whereas others were quite dissimilar.

Residents had similar responses when asked questions concerning: UTIs, imaging, frequency of surveillance and monitoring of kidney function. All residents agreed that treatment of asymptomatic UTIs in patients with indwelling Foley catheters and patients who performed clean intermittent catheterization was not indicated. This reflects previous guideline statements. ${ }^{4,5}$ Most residents would treat, both male and female, symptomatic UTIs for 7 days. All residents would use ultrasound as their imaging modality of choice to monitor the upper urinary tract. They would perform routine clinic follow-up annually, monitor kidney function annually with serum creatinine, monitor the upper urinary tracts annually and perform surveillance urodynamics on an as needed basis. Residents encountered high SCI patients in a wide variety of clinical settings however a third of residents had only encountered high SCI patients as hospital inpatients. There was also no consensus amongst residents regarding a few questions, specifically: choice of antibiotic for symptomatic UTIs, how frequently they would perform surveillance cystoscopy and how comfortable they were managing high SCI patients.

It is reassuring that graduating urology residents had very similar management algorithms for the stable high SCI patient, even in the setting of varied clinical exposure. The preference for chief urology residents to perform urodynamics on an as needed basis aligns with the European guidelines. ${ }^{8}$ It also is consistent with the prior systematic review by Cameron et al, which showed a lack of data in support for routine urodynamics. ${ }^{9}$ This systematic review of six published articles suggested routine urodynamics was warranted however there was no consistent data regarding at what frequency this should be performed. ${ }^{9}$ Most urology residents stated they would monitor renal function on a regular basis demonstrating a decision to avoid renal function deterioration. Serum creatinine, the laboratory test most chosen by urology residents, however has limitations in the SCI population. Some would recommend more accurate tests like nuclear medicine GFR or 24-hour creatinine clearance for this patient population. ${ }^{10}$ Previous research by MacDiarmid et al, comparing creatinine clearance with serum creatinine in the SCI population, found $31 \%$ of patients had a decline in creatinine clearance with a corresponding normal serum creatinine. ${ }^{10}$ The results of these studies however do not always coincide with the realities of the day-to-day urology practice. Although there was no consensus on how frequently urology residents would perform routine cystoscopy, it was clear most residents, $82 \%$, favoured routine cystoscopy compared to an as needed basis. Regular cystoscopy to avoid overlooking squamous cell carcinoma of the bladder in patients with indwelling Foley catheters is a recognizable concern for residents. However previous research has shown that, with a high index of suspicion with this population, it is unclear what screening interval should be followed for surveillance protocols. ${ }^{11}$ 
The potential lack of confidence or uncertainty described by chief urology residents with managing this patient population is of interest. Without a foundation of learning that provides trainees with the conviction to make consistent patient care decisions, there is a question of whether patients will be managed consistently across the country. Whether the same Royal College certified urology graduate will make equivalent management decisions for their next high SCI patient is a question for future research.

CBD integration is being implemented at all academic urology programs in Canada. With the introduction of CBD there is an opportunity to address confident, consistent management decisions made by chief urology residents with the structure of CBD allowing for specific concepts to be addressed and highlight gaps in knowledge. Deficits within the urology residents' training, whether that be managing a pregnant patient with ureteric calculi, navigating the steps of a cystectomy or managing a stable SCI patient in an outpatient setting, CBD has the advantage of identifying these gaps and establishes a protocol to address them prior to completion of their urology program ${ }^{6}$. Academic urology programs can ensure, with the aid of the CBD platform, that urology residents gain experience in managing this patient population and develop confidence making clinical decisions.

As chief resident opinions likely reflect the practice patterns of the centre where they trained, it can be assumed that there is a reasonable consensus on management of patients with SCI at the various academic institutions in Canada. Once published, the CUA guideline will provide clarification for the practicing urologist and urology residents.

This research is limited to the small sample size, gathering just over 30 subjects for the academic year. Although this figure does include a complete sampling of chief urology residents nationwide, it does not describe if these responses are common amongst graduating urology resident cohorts or outliers of a single year. This information also does not allow for quantitative analysis but descriptive data based on the candidates' responses. Whether the responses chief urology residents made would be similar one, five or ten years into their practice also is not available.

\section{Conclusion}

When surveyed during a preparatory Royal College examination, chief urology residents had similar management decisions for stable high SCI patients. Frequency of cystoscopy, as well as comfort in managing this patient population had varied responses amongst chief urology residents. This information could be used to help enhance and shape academic CBD urology programs. 


\section{References}

1. Noonan, VK, Fingas, M, Farry, A, et al. Incidence and prevalence of spinal cord injury in Canada: A national perspective. Neuroepidemiology 2012;38:219-226. https://doi.org/10.1159/000336014

2. Weld, KJ, Dmochowski, RR. Effect of bladder management on urological complications in spinal cord injured patients. J Urol 2000:163;768-72. https://doi.org/10.1016/S00225347(05)67800-7

3. Defade, BP, Kennelly, $\mathrm{M}$ and Deem, S. Urological Care of the Neurologically Impaired Patient in the Outpatient Setting. AUA update series 2011: Volume 30; Lesson 4;33-40.

4. Abrams, P, Agarwal, M, Drake, M, et al. A proposed guideline for the urological management of patients with spinal cord injury. BJU International 2008:101;989-94. https://doi.org/10.1111/j.1464-410X.2008.07457.x

5. Blok, BFM, Karsenty, G, Corcos, J. Urological surveillance and management of patients with neurogenic bladder: Results of a survey among practicing urologists in Canada. Can. J. Urol 2006:13;3239-43.

6. The Royal College of Physicians and Surgeons of Canada :: Competence by Design: the rationale for change. Available at: http://www.royalcollege.ca/rcsite/cbd/rationale-whycbd-e. (Accessed: 31st May 2018)

7. $\quad$ CBD Urology Competencies 2018. http://www.royalcollege.ca/cs/groups/public/documents/document/ltaw/mtc2/ edisp/rcp00176036.pdf

8. Stöhrer, M, Blok, B, Castro-Diaz, D, et al. EAU guidelines on neurogenic lower urinary tract dysfunction. Eur Urol 2009;56:81-8.

9. Cameron, AP, Rodriguez, GM, Schomer, KG. Systematic review of urological followup after spinal cord injury. J. Urol 2012;187,391-7.

10. MacDiarmid, S, McIntyre, WJ, Anthony, A, et al. Monitoring of renal function in patients with spinal cord injury. BJU Int 2000;85,1014-8.

11. Welk, B, McIntyre, A, Teasell, R, et al. Bladder cancer in individuals with spinal cord injury. Spinal Cord 2013;51,516-21. 
Figures and Tables

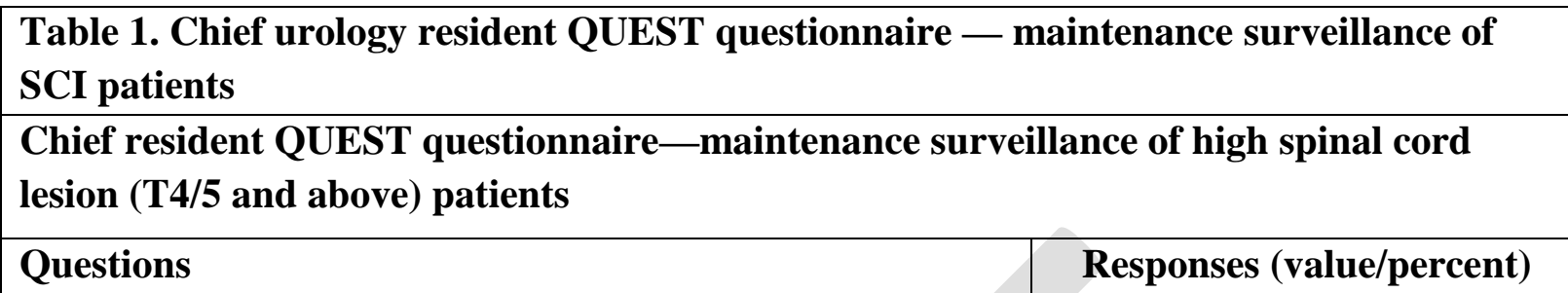

1. Where did you encounter high spinal cord injured patients during residency (check all that apply)?

\begin{tabular}{|l|c|c|}
\hline a. Rehabilitation centre (only) & 4 & 12 \\
b. Single practitioner clinic (only) & 2 & 6 \\
c. Hospital inpatients only (only) & 11 & 33 \\
d. Multidisciplinary clinic (only) & 0 & 0 \\
e. Rehabilitation centre + single practitioner + & 2 & 6 \\
f. hospital inpatients & 2 & \multicolumn{2}{|c|}{6} \\
g. Single practitioner + hospital inpatients & 7 & 21 \\
h. Single practitioner + multidisciplinary clinic & 2 & 6 \\
i. Hospital inpatients + multidisciplinary clinic & 2 & 6 \\
j. Rehabilitation centre + single practitioner clinic + & 1 & 3 \\
\hline hospital inpatients + multidisciplinary clinic & & \multicolumn{2}{|c|}{} \\
\hline
\end{tabular}

2. Do you treat asymptomatic urinary tract infections in patients with indwelling Foley catheters?

\begin{tabular}{|l|c|c|}
\hline a. Yes & 0 & \\
b. No & 33 & 100 \\
\hline
\end{tabular}

3. Do you treat asymptomatic urinary tract infections in patients who perform self, intermittent catheterization?
a. Yes
b. No
0 33
100

4. How many days do you treat symptomatic urinary tract infections in women with neurogenic voiding dysfunction of any etiology?
a. Single day
b. 3
c. 7
d. 10
e. 14

\begin{tabular}{|c|c|}
\hline 0 & 0 \\
8 & 24 \\
21 & 64 \\
3 & 9 \\
1 & 3 \\
\hline
\end{tabular}

5. How many days do you treat symptomatic urinary tract infections in men with 


\begin{tabular}{|l|c|c|}
\hline \multicolumn{3}{|l|}{ neurogenic voiding dysfunction of any etiology? } \\
\hline a. Single day & 0 & 0 \\
b. 3 & 3 & 9 \\
c. 7 & 22 & 67 \\
d. 10 & 5 & 15 \\
e. 14 & 3 & 9 \\
\hline 6. Which antibiotic do you prescribe when treating a urinary tract infection in a \\
symptomatic, afebrile patient prior to culture results being available? \\
\hline a. Sulfa/ sulfa-trimethoprim & 15 & 45 \\
b. Fluoroquinolone & 18 & 55 \\
c. Parenteral aminoglycoside & 0 & 0 \\
d. Ampicillin or another penicillin derivative & 0 & 0 \\
e. Cephalosporin & 0 & 0 \\
\hline
\end{tabular}

7. How frequently do you perform routine clinic follow-up in stable patients with neurogenic voiding dysfunction?
a. q 6 months
b. q 12 months

\begin{tabular}{l|l}
7 & 21 \\
7 & 73
\end{tabular}
c. q 24 months
d. No routine schedule - on an as needed basis
24
73
1
3

13

8. Which test do you use to monitor kidney function?
a. Serum creatinine
b. Creatinine clearance
c. Other

\begin{tabular}{c|c}
\hline 24 & 73 \\
7 & 21 \\
2 & 6
\end{tabular}

9. How frequently do you monitor kidney function in stable patients with neurogenic voiding dysfunction?
a. q 6 months
b. q12 months
c. q 24 months
d. Less frequently
e. No routine schedule - on an as needed basis

\begin{tabular}{c|c}
\hline 3 & 9 \\
28 & 85 \\
2 & 6 \\
0 & 0 \\
0 & 0
\end{tabular}

10. Which imaging modality do you use to monitor upper urinary tracts in stable patients with neurogenic voiding dysfunction?
a. Ultrasound
b. CT
c. Renal scan
d. None

\begin{tabular}{c|c}
33 & 100 \\
0 & 0 \\
0 & 0 \\
0 & 0
\end{tabular}

11. How frequently do you monitor upper urinary tracts in stable patients with neurogenic voiding dysfunction? 


\begin{tabular}{|l|c|c|}
\hline a. q 6 months & 0 & 0 \\
b. q 12 months & 23 & 70 \\
c. q 24 months & 8 & 24 \\
d. Less frequently & 0 & 0 \\
e. No routine schedule - on an as needed basis & 2 & 6 \\
\hline 12. How frequently do you perform surveillance urodynamic testing in stable patients with \\
neurogenic voiding dysfunction? & 3 & 9 \\
\hline a. q 12 months & 3 & 9 \\
b. q 24 months & 9 & 27 \\
c. Less frequently & 18 & 55 \\
\hline d. No routine schedule-on an as needed basis & 10 & 30 \\
\hline 13. How frequently do you perform surveillance cystoscopy in stable patients with \\
neurogenic voiding dysfunction and long-term, indwelling Foley catheters? \\
\hline a. q 12 months & 7 & 21 \\
b. q 24 months & 10 & 30 \\
c. Less frequently & 6 & 18 \\
\hline d. No routine schedule - on an as needed basis & 12 & 3 \\
\hline 14. How comfortable are you managing patients with high spinal cord injuries (T4/5 and \\
above)?
\end{tabular}

CTL computed tomography; q: every. 